\title{
Educomunicación e interculturalidad a partir de la gestión educativa con la radio
}

\author{
Educommunication and interculturality \\ from educational management with radio
}

\author{
Fernando Carias-Pérez \\ Universidad de los Lagos, Chile \\ fernando.carias@ulagos.cl \\ https://orcid.org/0000-0002-6955-6125 \\ Isidro Marín-Gutiérrez \\ Universidad de Sevilla, España \\ imgutierrez@us.es \\ https://orcid.org/0000-0002-6858-0983 \\ Ángel Hernando-Gómez \\ Universidad de Huelva, España \\ angel.hernando@dpsi.uhu.es \\ https://orcid.org/0000-0002-6414-5415
}

Recibido: 01/04/2021 Revisado: 26/04/2021 Aceptado: 11/05/2021 Publicado: 01/09/2021

\section{Resumen}

El presente artículo propone establecer criterios que permitan acoplar dos áreas disciplinares que pudieran parecer antagónicas pero que poseen características para articularse. En primer lugar, se plantean caracterizaciones de la educomunicación y la interculturalidad, razonadas con base en el rol que desde las ciencias sociales ocupan como áreas del conocimiento, para posteriormente ofrecer orientaciones sobre cómo pueden conectarse e interactuar para ponerse al servicio de proyectos e iniciativas de gestión educativa que vinculen a la educación, la comunicación y la cultura, específicamente desde el uso de la radio educativa en escuelas rurales del sur de Chile, las cuales poseen estudiantes de origen mapuche-huilliche. La perspectiva metodológica es de carácter cualitativa, hecha evidente mediante una revisión bibliográfica desde un compendio de material referencial, con la intención de generar el marco teórico de una investigación doctoral con foco en prácticas educomunicativas en contextos interculturales, así como datos obtenidos a partir de un trabajo de campo y entrevistas semiestructuradas a informantes claves. El artículo evidencia cómo pueden generarse propuestas que redunden en hacer más efectivos los procesos formativos de los estudiantes en contextos de interculturalidad, así como el fortalecimiento identitario y revitalización cultural para favorecer la construcción de sociedades democráticas y una cultura de paz, teniendo como dínamo los fenómenos que emanan desde la comunicación.

\section{Palabras clave}

Educomunicación, interculturalidad, radio, Chile, cualitativa, entrevistas, estudiantes, cultura.

Forma sugerida de citar: Carias-Pérez, F.I., Marín-Gutiérrez, I., \& Hernando-Gómez, A. (2021). Educomunicación e interculturalidad a partir de la gestión educativa con la radio. Universitas-XXI, 35, pp. 39-60. https://doi.org/10.17163/uni.n35.2021.02 


\begin{abstract}
This article proposes to establish criteria that allow the coupling of two disciplinary areas that may seem antagonistic but that, nevertheless, have characteristics to be articulated. In the first place, characterizations of educommunication and interculturality are proposed, reasoned on the basis of the role that the social sciences occupy as areas of knowledge, to later offer guidance on how they can connect and interact to put themselves at the service of management projects and initiatives that link education, communication and culture, specifically from the use of educational radio in rural schools in southern Chile, which have students of mapuche-huilliche origin. The methodological perspective is qualitative in nature, made evident by a bibliographic review from a compendium of reference material, with the intention of generating the theoretical framework of a doctoral research focused on educommunicative practices in intercultural contexts, as well as data obtained from intense field work and semi-structured interviews with key informants. The article shows how proposals can be generated that result in making the formative processes of students more effective in contexts of interculturality, as well as the strengthening of identity and cultural revitalization to favor the construction of democratic societies and a culture of peace, having as dynamics the phenomena emanating from communication.
\end{abstract}

\title{
Keywords
}

Educommunication, interculturality, radio, Chile, qualitative, interviews, students, culture.

\section{Introducción}

La educomunicación es un área del conocimiento que se encuentra en construcción (de Oliveira Soares, 2011). Su puesta en práctica suele ser dialógica y se vincula generalmente con otras disciplinas. Esto hace que las definiciones del concepto sean muy dispares. Según Roberto Aparici (2010) es Latinoamérica el sitio donde se ha aportado más a la educomunicación desde la visión teórica y epistemológica.

De hecho, podríamos afirmar que la historia de la educomunicación se remonta a la construcción de lo que es hoy la realidad social latinoamericana con sus luces y sombras (Lotero-Echeverri et al., 2019). Esto supuso reflexiones y discusiones en el mundo académico que se ha aproximado a la educomunicación para generar diálogos interdisciplinarios que permitan el estudio y comprensión de fenómenos de distinta índole. En esta línea, la 
educomunicación se plantea como dialógica, teniendo la posibilidad de vincularse con otras áreas del conocimiento, generando importantes espacios para el diálogo de saberes entre diferentes modos de ver y comprender la realidad. Una de estas áreas o campos del saber, es la interculturalidad (Collado-Ruano et al., 2020).

Por otro lado, la interculturalidad ha sido en los últimos años uno de los temas más recurrentes en Latinoamérica y Europa (Rodríguez-Pastene et al., 2020). Por mucho, resulta complejo poder caracterizarla pues las posturas y reflexiones han tomado caminos disímiles según la perspectiva científico-social desde donde se plantee. La interculturalidad se ha entendido como paradigma epistemológico, se considera principio fundamental para la comunicación en contextos multiculturales y también un enfoque para la elaboración de políticas públicas, utopía, entre otros (González \& Rodríguez, 2019).

La interculturalidad como concepto polisémico puede ser comprendido desde diversas epistemologías, traducciones culturales y campos temáticos, pero a su vez como un proyecto social vinculado en muchas ocasiones con la educación, una dimensión que surge como interés por hacer dialogar a todas las culturas que se encuentran dentro de la sociedad. En el mundo educativo se denominó a la educación intercultural como una visión reformadora del quehacer pedagógico que responde sobre la diversidad cultural en el mundo moderno (Arispe, 2020).

Entendido así, la educomunicación y la interculturalidad se presentan como disciplinas y espacios epistémicos propicios para el abordaje de situaciones en diferentes contextos, entre ellos aquellos relacionados a la multiculturalidad o a los fenómenos contemporáneos que plantean el diálogo comunicativo (simétrico o asimétrico) entre culturas, pudiendo ofrecer espacios para la comprensión y resolución de conflictos dentro de la diversidad y los distintos ámbitos de la vida y la cotidianidad, siempre desde una perspectiva que involucre a la educación y la comunicación, así como las interfaces que se generan desde sus tensiones (de Oliveira Soares, 2009). Planteamos una caracterización de ambas a partir de sus principios fundamentales, proponiendo analogías para identificar cómo pueden conectarse y generar orientaciones para una puesta en práctica o en su defecto, para situarlas en espacios de concreción real.

La radio educativa es aquel espacio formativo, formal e informal, donde se utiliza la lógica de la radiodifusión con el fin de utilizarlos en las técnicas 
de enseñanza y aprendizaje (Laor, 2020). La radio educativa se basa en generar y producir, junto a los estudiantes, contendidos radiofónicos en pequeños formatos como cápsulas informativas, seriales o micronoticieros, para su difusión interna o externa (en el establecimiento educacional o a través de alguna emisora con señal abierta). La radio representa y justifica la perspectiva educomunicativa que buscamos evidenciar.

La trama involucra a la educación, a la comunicación y a la cultura como ejes del discurso y se evidencian sobre la base de iniciativas en contextos escolares de instituciones del sur de Chile, donde se ha utilizado la radio educativa como mediadora de procesos vinculados a la revitalización cultural en estudiantes de origen mapuche-huilliche, teniendo a la educomunicación como artefacto para gestionar buenas prácticas educativas.

\section{Marco teórico}

\section{Educomunicación, perspectivas teóricas para su caracterización}

Desde la Declaración Universal de Derechos Humanos (1948), la educación, comunicación y la participación se consideran derechos humanos fundamentales. Este marco nos permite abordar y de alguna manera, caracterizar el concepto de educomunicación, como un constructo vivo que dialoga con los contextos en los cuales se desenvuelve (Marín-Gutiérrez et al., 2020). La educomunicación para Aparici es:

El conocimiento de los múltiples lenguajes y medios por los que se realiza la comunicación personal, grupal y social. Abarca también la formación del sentido crítico, inteligente, frente a los procesos comunicativos y sus mensajes para descubrir los valores culturales propios y la verdad. (Aparici, 2010, p. 9)

Esto puede traducirse en que la educomunicación se construye en función de las distintas definiciones. Oliveira Soares (2011) afirma que la educomunicación es una práctica social educativa asignada a elementos de la modernidad como son los medios de comunicación y las TIC. Han surgido diferentes líneas temáticas a partir del término educomunicación para designar la relación entre los dos campos; educación en medios (vigente en Eu- 
ropa hasta la fecha) y lectura crítica de la comunicación (en uso en América Latina, entre 1970 y 1980), los cuales son anteriores al uso del neologismo educomunicación (Kaplún, 2006). Ello describe diferentes situaciones de la interfaz entre estos conceptos y abarcan diferentes aspectos de la relación entre educación y comunicación.

De acuerdo con Olimpia Mata (2010) la educomunicación se define como una educación "con", "para" y "en" la comunicación. La educación "con" la comunicación, que hace referencia al uso de los medios para su análisis. La clave para comprender esta idea es "el educarse aprendiendo a leer de forma crítica los mensajes que nos transmiten los medios de comunicación" (Mata, 2010, p. 2).

La educación "para" la comunicación pretende llegar a una relación entre emisor y receptor. La educación "en” la comunicación se entiende a través de las TIC. Se utilizarán los medios de comunicación en los procesos de enseñanza-aprendizaje.

Otra definición que nos sitúa en una caracterización de la educomunicación, es aquella que ofrece Hernández-Díaz (2007), que escribe que la educomunicación "es un campo de carácter interdisciplinario que abreva fundamentalmente de la ciencia de la educación y la comunicación, así como los aportes teóricos de las ciencias sociales en general" (p. 68).

Así mismo, la educomunicación como área del conocimiento, se concretiza y se hace tangible desde su puesta en práctica. A partir de lo que hace y genera en la realidad. A través de proyectos o iniciativas en forma de planes y programas socio-educativos y socio-culturales, sean estos desde instituciones públicas, privadas o incluso, sobre la base de investigaciones como la que aquí se presenta. Por lo que estas instancias generan insumos para el constructo epistemológico de la disciplina, siendo imperativa la necesidad de espacios de sedimentación del saber en procura de nutrir un campo en constante generación de conocimientos, aportando al logro de sociedades democráticas (Muñoz-Borja et al., 2021).

Tanto desde un punto de vista teórico como práctico hoy no podemos hablar de comunicación y educación como un ámbito cerrado. HidalgoSantomaro y Gamboa-Chiriboga (2012) señalan que la educomunicación debe ser trabajado desde un ángulo interdisciplinario, tanto epistemológico, pedagógico y social. Por una parte, la educación y, por otro lado, la comunicación. 
Cuando nos referimos a la educomunicación nos remitimos a una disciplina o área del conocimiento en proceso de consolidación, que se discute desde distintas perspectivas, planteamientos y escenarios, pero que sobre todo requiere de praxis para concretizarse (Andrade-Martínez, 2020).

Más allá de los conceptos y la episteme que se genera a partir de ella, hay que comprender a la educomunicación como parte de los procesos de un mundo dinámico e interconectado, de cambios rápidos generados por las presiones que ejerce la globalización. En este contexto posmoderno será importante cualquier perspectiva disciplinar que plantee una redefinición del sujeto social y de su contexto sociocultural, por lo cual la educomunicación es una vía posible (Moreira, 2020).

\section{Interculturalidad}

El término interculturalidad suele ser complejo de caracterizar. No existe un marco de referencia desarrollado sobre la interculturalidad y su naturaleza como paradigma. Generar un concepto supone tomar diferentes caminos y posturas epistemológicas, las cuales se vinculan con variadas disciplinas y perspectivas que se desprenden de las ciencias sociales y áreas del conocimiento afines. También se suele caracterizar el término a partir de los contextos y realidades en donde se aplica. Lo que supone que puede ser entendido de diferentes maneras, siendo por ejemplo que, no es lo mismo lo que pudiera entenderse por interculturalidad en Europa con respecto, por ejemplo, a Latinoamérica (Di Caudo et al., 2016).

El concepto hace referencia explícita a los contactos que se dan entre personas de distintas culturas. Esta suele ser la acepción más común pues, en esencia, la humanidad es y ha sido siempre intercultural. Néstor García Canclini (2004) delimita el término e intenta reconocer las diferencias, desigualdades y conexiones que se dan en todo este proceso, afirmando que "Adoptar una perspectiva intercultural proporciona ventajas epistemológicas y de equilibrio descriptivo e interpretativo, logrando concebir políticas de la diferencia" (p. 13).

Entendido así es cómo se va modelando en los discursos la idea de la interculturalidad. Sin embargo, es importante no tratar de entender la interculturalidad como un espacio o "lugar" ideal para el desarrollo armónico entre las culturas, lo cual es válido pero tiene el problema de que no se investigue 
en el tema del conflicto intercultural y no se ocupe del sentido político del concepto, lo que problematizaría aún más su caracterización, así como también colocaría a la interculturalidad en un discurso utópico y hueco, incapaz de zanjar las brechas de la desigualdad (Rodríguez-Pastene et al., 2020).

Hasta aquí la interculturalidad se va configurando desde dos perspectivas esenciales, una reconciliadora, menos pragmática, más "blanda" y otra vinculada a un matiz más desafiante, que problematiza en profundidad e invita a miradas complejas de los contextos en donde se desarrolla. Tubino (2005) se refiere a los siguientes conceptos: el interculturalismo funcional (o interculturalismo neoliberal) y el interculturalismo crítico (o interculturalismo liberador). El primero, se suscribe a una postura que se vale del diálogo, pero de una manera asimétrica, postulando discursos que utilizan la interculturalidad, como herramienta para hacer impalpable y evidentes los grandes problemas de fondo que poseen las sociedades modernas, tales como; la xenofobia, el racismo, la desigualdad de género, la pobreza, las injusticias sociales o la inequidad, mediante el desconocimiento y en algunos casos la supresión de las propias culturas. Es una interculturalidad estéril que responde a una forma y un paradigma de sociedad fuertemente deshumanizado. Tubino (2005) bien lo sintetiza en estas líneas: "Se trata de un discurso y de una praxis de la interculturalidad que es funcional al Estado nacional y al sistema socioeconómico vigente" (p. 33).

En tanto el interculturalismo crítico (o interculturalismo liberador), asume una posición más comprometida con las sociedades, entendiendo la interculturalidad como un espacio dialógico para la solución de los conflictos y las desigualdades, apostando a la transformación real de las estructuras, respetando las diferencias y sobre todo entendiendo que existen "otras" formas de entender el mundo, "otros" saberes, "otros" constructos. El interculturalismo crítico es, en esencia, una propuesta ético-política de cambio sustancial (Tubino, 2005).

En la discusión de las diferencias entre multiculturalismo e interculturalidad, recurrente en las caracterizaciones de ambas, Dietz (2012) propone para su comprensión, un modelo de gestión de la diversidad que se basa en el reconocimiento de la diferencia y la otredad, a partir de paradigmas (lo multicultural y lo intercultural) que hacen énfasis en la interacción e interrelaciones que se generan entre miembros de diferentes grupos y franjas sociales. 
Tabla 1

Modelo de gestión de la diversidad

\begin{tabular}{|c|l|l|}
\hline \multicolumn{1}{|c|}{ Plano fáctico } & \multicolumn{1}{|c|}{ Multiculturalidad } & \multicolumn{1}{c|}{ Interculturalidad } \\
\hline = lo que es & $\begin{array}{l}\text { Diversidad cultural, } \\
\text { Lingǘstica, religiosa. }\end{array}$ & $\begin{array}{l}\text { Relaciones interétnicas, } \\
\text { interlingǘsticas, interreligiosas. }\end{array}$ \\
\hline Plano normativo & \multicolumn{1}{|c|}{ Multiculturalismo } & \multicolumn{1}{c|}{ Interculturalismo } \\
\hline = lo que debería ser & $\begin{array}{l}\text { Reconocimiento de la } \\
\text { diferencia } \\
\text { 1. Principio de igualdad } \\
\text { 2. Principio de diferencia }\end{array}$ & $\begin{array}{l}\text { Convivencia en la diversidad } \\
\text { 1. Principio de igualdad } \\
\text { 2. Principio de diferencia } \\
\text { 3. Principio de interacción } \\
\text { positiva }\end{array}$ \\
\hline
\end{tabular}

Fuente: Elaboración propia con base en Dietz (2012).

La interculturalidad utilizada como metodología es una interdisciplina que nos va a ayudar a analizar las interacciones con distintas culturas. Es una forma de vida en la que se va creando una toma de posición ética a favor de la convivencia con las diferencias (Hernández-Reyna \& Castillo-Cocom, 2021).

\section{Educación con perspectiva intercultural}

Actualmente existe un amplio debate académico, pedagógico, social, pero sobre todo político con respecto a la educación intercultural. Por un lado, proliferan los programas y currículos, no siempre exitosos, que tratan de hacer frente a la diversidad cultural que es más evidente en las clases de cada uno de los niveles educativos en todo el mundo. Por otro lado, las divergencias con respecto a un paradigma de similitudes medianamente armónicas, sobre la educación intercultural, no termina de consolidarse entre quienes estudian esta área de la pedagogía contemporánea. Lo último no resultaría tan negativo, si no fuera por la enorme cantidad de estudios empíricos que tratan de caracterizar a la educación intercultural, no sin antes generar líneas y miradas de toda índole, lo que dificulta hacerla concreta en un concepto en común.

Así lo afirma Dietz (2012) cuando afirma que no se tiene una definición aceptada globalmente de lo que es la educación multicultural o intercultural. 
El concepto se adhiere a una perspectiva de educación intercultural en vínculo con las culturas indígenas o, mejor dicho, una educación intercultural con foco indígena, lo que a su vez remite al campo de la educación intercultural bilingüe (Granda-Merchán, 2016). Esta es una línea educativa que se ha desarrollado, sobre todo, en Latinoamérica desde hace 50 años hasta nuestros días (Gajardo-Carvajal \& Mondaca-Rojas, 2020).

Los proyectos de educación intercultural ofrecen nuevos espacios de comunicación entre las personas, generando zonas de reciprocidad y diálogo contribuyendo a cambiar la idea de enclaustrar a las culturas como reservas culturales (Núñez-Ruiz et al., 2020). En este sentido, afirman Escarbajal de Haro et al. (2007) que "no se debe renunciar a la propia identidad, pero tampoco esa identidad particular puede impedir la convivencia en la diversidad" (p. 27). Las sociedades occidentales actuales tienen como características, la diversidad de formas de socialización, de obtener distintas culturas, de crear su identidad personal, de adquirir distintas lenguas, de formas de pensar y de relacionarse con los otros. Todos estos elementos que se evidencian en los espacios escolares. El objetivo de los colegios es integrar a las personas en la sociedad cuestión que desde una perspectiva intercultural puede generarse (Delbury, 2020).

Existen algunos preceptos para aproximarnos y comprender la educación intercultural, como son buscar enfoques complejos, holísticos y multidisciplinares. También utilizar enfoques multidimensionales. Otro es el compromiso en la construcción de una sociedad democrática y compromiso con los derechos humanos. También existe un máximo respeto a las minorías culturales (Ari \& Stöckli, 2021).

La educación intercultural debe favorecer a promover la riqueza y la diversidad que ha caracterizado a la humanidad a través de la historia, aportando en la comprensión de que no existe una sola verdad, "que la vida social con la que nos identificamos no es la única, sino una de tantas posibles, que el modelo económico y social de Occidente no es, ni global ni necesariamente, exportable al resto del mundo, ni de obligada asunción por otras culturas" (Escarbajal de Haro et al., 2007, p. 54).

\section{Metodología}

El estudio que da origen a este artículo se enmarca en su diseño y métodos dentro de una perspectiva cualitativa (Gibbs, 2012), ya que los objeti- 
vos de la investigación apuntaban hacia un análisis descriptivo del rol de la radio educativa en contextos de educación intercultural. Se trata en su conjunto de un estudio de caso (Simons, 2011) enfocado en tres escuelas rurales del sur de Chile, específicamente ubicadas en la Región de Los Lagos (X Región), Provincia de Osorno, comunas de San Pablo y San Juan de la Costa, las cuales utilizan los medios de comunicación social, específicamente la radio, como recurso educativo. La investigación se desarrolla desde un proceso de exploración caracterizado por el estudio en profundidad de ciertos fenómenos, los cuales pueden ser estudiados a partir de entidades sociales o entidades educativas (Sabariego et al., 2019), con la especificidad de poseer un carácter exploratorio y descriptivo ya que se detallan situaciones, eventos y procesos interactivos y experiencias únicas.

La propuesta metodológica se justifica en función de dos momentos que son dialógicos y se complementan para así evidenciar los resultados y conclusiones que presentamos. El primero, consta de una revisión bibliográfica y de material documental relacionada a los temas de educomunicación, interculturalidad, comunicación y ciencias sociales, donde se ha construido un discurso en procura de caracterizar las dos disciplinas que componen y se acoplan sobre la base de la propuesta, el cual es apéndice de una investigación aún más extensa sobre los temas de radio, educomunicación e interculturalidad a partir de prácticas educomunicativas en escuelas rurales del sur de Chile. Hemos acotado y resumido parte de la información que compone el marco teórico del estudio de origen y hemos traído aquellas ideas y planteamientos que nos parecen relevantes de cara a los propósitos del ideario de este escrito.

El segundo argumento metodológico, consistió en cruzar toda aquella información obtenida a través del trabajo de campo mediante la observación participante realizada in situ y el diario de campo obtenido con base a las interacciones. Se generó un grupo de 25 entrevistas efectuadas a informantes claves dentro de la investigación. Entre ellos estudiantes que participan de los talleres de radio en las escuelas participantes de la investigación, apoderados, personal administrativo y profesores, así como personas involucradas con las temáticas en estudio: investigadores, especialistas en comunicación, medios y educación o periodistas. Se concretó un eslabón esencial de esta cadena; el cruce de la información, el cual generó el análisis y resultados que es producto de la conjugación de todos los elementos antes descritos.

El proceso de revisión bibliográfica se ha realizado a partir de tres pasos: la búsqueda de la información; la organización de la información; y el estudio de la información. 
La búsqueda de la información comenzó a través de la revisión bibliográfica que consistió en recopilar material referencial como libros, revistas de divulgación o investigación científica, tesis de maestría y doctorado, sitios web, material audiovisual, entre otros, vinculados a la temática de estudio. Supuso un proceso lento, meticuloso y selectivo que implicó leer y seleccionar aquellos aportes teóricos de nuestro interés, desde las fuentes de información consultadas. Se delimitó la búsqueda en función de las preguntas u objetivos de la investigación, para luego realizar una selección depurada de aquellos aportes que consideramos relevantes. Dada la gran cantidad de material e información consultada hemos tomado aquellas referencias que consideramos esenciales y validan las propuestas de nuestras ideas.

Sobre la organización del material se estableció un criterio de sistematización (Pérez-Serrano, 2016) según el uso de Zotero, herramienta informática que permitió ir ordenando la información de los materiales referenciales por título, autor, tipo de documento y aporte o idea que nos interesó rescatar. Se discriminó referencias relevantes en detrimento de aquellas que fueron consideradas de segundo orden. Se generó un repositorio simple a partir de un archivo informático, donde se fueron acumulando aquellos enunciados que pudieran ser utilizados como citas dentro del texto del marco teórico para luego junto a los argumentos del cruce de otros datos, poder armar la trama de la investigación y los argumentos que sustentan este escrito.

Para completar el ciclo se realizó el análisis de la información. Para ello se generaron descriptores primarios y códigos, identificados en los grupos temáticos más significativos para hacer un cotejo de co-citación de autores y de coocurrencia que permitió identificar aquellas ideas que resultaran redundantes, pero al mismo tiempo, importantes.

Dentro de las estrategias utilizadas con base en la lógica de la investigación cualitativa, se trabajó la observación participante y las entrevistas abiertas o semiestructuradas a informantes claves (McMillan \& Schumacher, 2005), partiendo de un plan de trabajo que condujo a más de tres años de contactos y visitas a las escuelas, así como encuentros de manera presencial y virtual con los informantes, especialmente durante el 2020, momento de crisis sanitaria a nivel mundial debido a la pandemia por coronavirus.

Una vez agrupados todos los datos y evidencias, se comenzó el proceso de fragmentación de la información, momento en el cual se generaron desde los objetivos de la investigación categorías generales, de las cuales a su vez se desprendieron unidades de análisis. Este proceso se realizó asistido 
por el software Atlas.ti, en el cual se conjugaron también la teoría, material audiovisual como audios de los programas de radio realizados en las escuelas e información complementaria como guiones, manuales de producción radiofónica, entre otros. Todo el proceso descrito fue dialógico y se caracterizó por cierto grado de atemporalidad y fragmentación desde su perspectiva metodológica.

\section{Resultados}

\section{Educomunicación e interculturalidad, pistas para su conexión}

Para generar aproximaciones interdisciplinares entre la educomunicación y la interculturalidad, debemos situarnos en el caso específico del trabajo que han realizado escuelas rurales del sur de Chile, específicamente en la provincia de Osorno, región de Los Lagos, donde se han utilizado iniciativas y proyectos vinculados a la radio educativa para generar espacios de revitalización cultural. En los colegios Misión San Juan y Quilacahuín, ambos pertenecientes a la Fundación Misiones de la Costa, se ha trabajado con los estudiantes, en la generación de contenidos radiales relacionados a la cultura mapuche huilliche, a partir de temas como la historia originaria del pueblo, sus tradiciones, mitos, rituales, celebraciones y especial énfasis en el aprendizaje y práctica del idioma propio; el chesungun.

La radio educativa podría caracterizarse como aquella que se encarga de generar contenidos que sirven con fines formativos. Han sido muchas las experiencias que se han generado en Latinoamérica, las cuales han estado esencialmente vinculadas a solventar problemas de cobertura que han tenido los sistemas educativos nacionales, relacionados a la incapacidad de poder llegar a lugares remotos o de difícil acceso, siendo la radio una herramienta eficaz para solventar, comunicacionalmente esos inconvenientes. Más allá de los currículos y los planes de estudio cerrados y formales, la radio puede, igualmente, cumplir una labor educativa desde espacios informales y no formales, generando desde sus propuestas una perspectiva que eduque desde las particularidades de los contextos y realidades donde acciona. Como medio de comunicación requiere ser entendida desde una lógica instrumental y técnica que determina incluso su funcionalidad desde una aplicación educativa, por lo que requiere tomar en cuenta una estructura de producción 
de objetos de aprendizaje o contenidos (Ferraretto \& Morgado, 2020). La radio propone elementos contundentes en la interfaz educación-comunicación, por lo que es utilizada en iniciativas y proyectos que involucran estas dos perspectivas.

Toda práctica educomunicativa es una dialéctica de la participación, una iniciativa que busca la construcción del conocimiento de manera colectiva, de forma horizontal, simétrica y recíproca (Barbas-Coslado \& Ortiz de Zárate, 2020). Se define como una acción facilitadora de los procesos que refuerzan aquellas competencias comunicacionales, mediáticas y relacionales para un mejor desenvolvimiento personal y social, generando espacios transformadores de los contextos donde se generan (Jackiw \& Haracemiv, 2021). Es una actividad que crea comunidad, tejido social a partir de la acción de comunicar, no solo de manera bidireccional, como habitualmente conocemos el proceso de la comunicación, sino también como una forma de expresar emociones, ideas, experiencias y saberes.

En el aula o en el contexto escolar, esto se evidencia como un proceso activo, dialógico, como un acto comunicativo, en donde los estudiantes construyen su conocimiento desde el desarrollo del pensamiento crítico, mediante actividades que se relacionan con situaciones del mundo real. De hecho, con sus propias realidades, permitiéndoles entender su rol social y personal, todo desde una perspectiva didáctica adecuada, que complemente los aprendizajes en cualquier área del conocimiento (Muñoz-Borja et al., 2021).

Evidenciamos las conexiones entre educomunicación e interculturalidad como una franja interfaz, que promueve acciones desde la escuela o fuera de ella, en procura de generar espacios dialógicos para una cultura de paz y la construcción de sociedades democráticas e interculturales, basadas en el reconocimiento del otro, teniendo como recurso para ello las TIC (Collado-Ruano et al., 2020). No se trata únicamente de un diálogo bidireccional, etéreo e infértil, se trata en esencia de un diálogo de saberes que busca comprender y contextualizar el conocimiento en todas sus dimensiones, permitiendo entender los problemas y necesidades que tiene la sociedad en su conjunto, mediante la reflexión y la discusión de sus actores y todos quienes la conforman.

Esta franja interfaz, como hemos denominado el lugar de encuentro entre educomunicación e interculturalidad, entendemos genera tres dimensiones que la concretizan. La primera es de carácter social y política y apunta hacia la participación de las personas y la creación de sociedades plurales. 
En esta dimensión son relevantes y primordiales aquellos elementos de carácter cultural que distinguen a grupos, colectivos o etnias. Aquí se manifiestan las conductas sociales y culturales en diferentes contextos. Prevalece aquí una revalorización de los derechos individuales y grupales (Derechos Humanos), resaltando las diferencias y la perspectiva de la otredad (Esquirol, 2005). Es una dimensión que permea al resto y se supedita a los paradigmas estructurales de la sociedad, incluidos aquellos de carácter económico y cultural. Aquí también se forjan espacios que plantean una crisis, en tanto la concepción del mundo actual, evidenciado en un cuasi consenso, que la modernidad ha generado esferas para la diferencia y los individuos crecen en un mundo en el que existen los valores comunes, lo cual en la realidad no es así, pues hoy más que nunca el pluralismo moderno socava el conocimiento de las particularidades y asume una perspectiva monocultural de la realidad.

La segunda se vincula con una dimensión educativa que busca elevar el conocimiento como un constructo social e interactivo, teniendo como objetivo formar y preparar a las personas para el protagonismo y la cooperación social. Aquí la educación debe ser entendida como un eslabón fundamental para consolidar y construir sociedades más democráticas, en detrimento de la idea histórica vinculada a modelos de desarrollo. Se comprende esta dimensión desde la perspectiva de la educación formal pero también debe salirse de ella y ceñirse al ámbito de lo no formal y lo informal, pues en esos espacios existen igualmente experiencias significativas que construyen ciudadanos activos, críticos y reflexivos. Se redimensiona el conocimiento, el cual debe ser entendido como una actividad social del hombre, donde no solo se vincula con ámbitos científicos, sino también con experiencias personales y vivenciales a partir del ver, el oír, el sentir, lo que desde la epistemología se denomina "Fenomenología del Conocimiento", donde se plantea esta dualidad, la cual por un lado está la conciencia o el sujeto del conocimiento y por el otro, el objeto, la realidad que se da al sujeto (Parra-Alvarracín, 2000). La dimensión educativa es fundamental en la construcción del entramado educomunicación-interculturalidad pues resulta un elemento nuclear dentro del análisis y la propuesta de este artículo.

Por último, una dimensión comunicacional que entiende los procesos comunicativos como dinámicos y críticos, donde las personas hagan del acto de comunicar un espacio para cuestionar, manifestar, defender y proponer sus deseos, necesidades y formas de transformar la realidad. Se propone un enfoque hacia los procesos de la escuela, desde la óptica de la comuni- 
cación, lo que supone una adecuada comprensión de los fenómenos y dinámicas de la realidad, de las comunidades y claro, la sociedad. Las formas en que se concretizan estas líneas, las proponemos a través de experiencias significativas desde el uso de la radio educativa en escuelas rurales del sur de Chile, específicamente en la revitalización de la cultural mapuche huilliche, enfocando el trabajo en el aprendizaje del chesungun, el cual busca potenciar no solo competencias comunicativas lingüísticas vinculadas a la cultura, sino también reforzar los aprendizajes desde la lectoescritura, la creatividad y el trabajo en equipo. El componente comunicacional acompaña los procesos de enseñanza y aprendizaje con una alta pertinencia cultural.

\section{Educomunicación con interculturalidad y viceversa}

Siendo la radio un medio de comunicación que aún en la actualidad llama la atención de las audiencias, a pesar de la consolidación de los medios digitales, esta sigue siendo utilizada como una potente herramienta para mediar en los procesos de enseñanza y aprendizaje. Tomaremos las experiencias de escuelas rurales al sur de Chile durante el 2020 y en el contexto de la pandemia, donde se ha utilizado la radio no solo como recurso para reforzar los aprendizajes y el trabajo en equipo, sino también como un dispositivo de comunicación efectiva ante el escaso acceso a Internet. Además de un instrumento para favorecer los procesos de revitalización de la cultura mapuche-huilliche, ofrecemos ahora, a manera de pistas enunciadas, formas y maneras en cómo pueden activarse procesos que involucren prácticas educomunicativas con el hecho intercultural.

La educomunicación, mediante su praxis a través de la radio, puede generar espacios dialógicos significativos entre las culturas, pudiendo ser un punto fértil y apropiado para la resolución de conflictos. En vínculo con los medios de comunicación y las TIC y sus mediaciones, ofrece formas de hacer efectiva y expansiva la expresión y voz de todos quienes se involucran en las dinámicas entre culturas (Franky \& Chiappe, 2018).

Desde las prácticas educomunicativas es posible generar iniciativas y proyectos para el resguardo y revitalización de "otras" culturas, utilizando instancias de producción y difusión de contenidos mediáticos, que pueden estar al servicio de los grupos, conglomerados o etnias para su consumo propio o, por el contrario, pueden servir para dar a conocer (hacia afuera) las 
costumbres y tradiciones propias de cierta cultura. Para el caso específico de las escuelas Misión San Juan y Quilacahuín, desde la radio educativa se generaron micros formativos didácticos para reforzar contenidos del currículo relacionados con la educación intercultural bilingüe, con el objetivo de que el alumnado conozca y se relacionen con la cultura mapuche-huilliche. Pero, al mismo tiempo, estas producciones radiales se utilicen como herramienta de transmisión de esa misma cultura una vez expuesta en los distintos medios de comunicación.

La interculturalidad debe ser entendida como un espacio de comunicación que exalta el diálogo de saberes y como tal, caben todas aquellas prácticas vinculadas a ella, incluso las que se sirven de todos los recursos que ofrecen las TIC y los medios de comunicación, entiéndase herramientas para la gestión, producción y difusión de contenidos.

En educomunicación se pueden generar iniciativas viables para la revitalización de los idiomas propios como el chesungun (mapuche-huilliche), el cual corre peligro de desaparecer debido al poco interés que tienen los jóvenes con respecto a mantener sus tradiciones lingüísticas. El trabajo desde la radio educativa ha evidenciado cómo pueden generarse instancias de aprendizaje del idioma a partir del bilingüismo y a través de contenidos en diferentes formatos, que permiten una adecuada aproximación al uso habitual y pertinente de ciertas formas de comunicación desde la cultura propia.

Las costumbres y tradiciones de los grupos minoritarios pueden, desde una perspectiva intercultural, ser representadas y mediatizadas no solo desde la idea de ser difundidas, sino también como documento y registro que resguarde la memoria colectiva, la identidad cultural y el patrimonio inmaterial de estos pueblos (Cebrián de la Serna, 2009).

La escuela resulta un espacio fundamental y fértil para que el binomio educomunicación e intercultural puedan conjugarse. En tal sentido, el piso y estructura que ofrece la educación intercultural bilingüe es idóneo para la realización de prácticas educomunicativas desde un sinfín de posibilidades.

Una perspectiva importante que también se desprende de la práctica educomunicativa, tiene que ver con la construcción de un criterio crítico y reflexivo de las TIC y los medios de comunicación mediante propuestas desde la escuela (Buckingham, 2003). En contextos interculturales puede utilizarse la educomunicación como recurso para que el alumnado acceda a la perspectiva que ofrece la alfabetización mediática e informacional como herramienta para la construcción de identidades en los espacios mediáticos. 
La puesta en práctica de estos criterios mediante la radio educativa ha evidenciado que esta ha venido conquistando una función mediadora en tanto procesos comunicacionales comunitarios que promueven la consolidación de las identidades culturales, sociales y políticas de las minorías, legitimando las formas de pensar y los significados, sirviendo, además no solo como una herramienta educativa sino también como instrumento para el desarrollo local (de la Noval-Bautista, 2018).

\section{Conclusiones}

La educomunicación y la interculturalidad poseen elementos que promueven positivamente su coexistencia, generando espacios, según el contexto, para la reafirmación de la identidad cultural y los procesos para alcanzar sociedades verdaderamente democráticas. Se trata de dos disciplinas que han tenido un desarrollo verosímil dado los innumerables problemas, conflictos y dinámicas que surgen en nuestras sociedades. Parte de las propuestas que esbozamos en este artículo es que, desde ambas disciplinas, las sociedades y los pueblos puedan reconocerse y puedan desarrollarse como agentes de cambio desde una perspectiva endógena, aprovechando la comunicación, los medios y los mensajes con foco en sus cotidianidades y sus contextos particulares, utilizando la educación como una herramienta que permita la generación de un pensamiento crítico y reflexivo del mundo y las realidades en las que vivimos.

Si bien la educomunicación hoy en día es un campo en constructo (de Oliveira- Soares, 2011), el cual no ha sido vinculado a los currículos nacionales a lo largo y ancho de Latinoamérica, cada día va ganado más espacios desde la educación no formal y a través de proyectos o instancia de gestión educativa que permiten involucrar la perspectiva educomunicativa como línea formativa dentro de la escuela e incluso, fuera de ella. La educomunicación cada vez más se afianza, gracias a soportes teóricos y científicos, como una disciplina que propone sólidas bases epistemológicas, desde las cuales es posible repensar y sintetizar el vínculo entre educación y comunicación. Esta disciplina puede a su vez, desde su praxis, generar proyectos que confronten y transformen la realidad desde propuestas relacionadas al buen vivir y el bienestar sociocultural, lo que sin lugar a dudas puede traducirse en las bases para una cultura de paz. 
Por otro lado, en tanto la interculturalidad, todos los modelos que tratan de explicarla convergen en la idea que, en esta se generan profundos procesos de carácter comunicativo que hacen dialogar a las culturas que se involucran, siendo hoy la discusión principal cómo se plantean estos "diálogos", los cuales se debaten entre lo simétrico y lo asimétrico, entre el respeto a la otredad y el desconocimiento peyorativo de las diferencias. Hay un elemento relacionado a lo intercultural y es el hecho per se de ser un acto comunicativo, lo que la convierte también en un campo interdisciplinario en el que confluyen enfoques psicológicos, lingüísticos, antropológicos y pedagógi$\cos$, lo que la vincula con la misma educomunicación. Desde esa perspectiva de la "comunicación intercultural", no solo se caracteriza por su función semántica, sino que también debe ser entendida desde una perspectiva de comprensión de códigos que se relacionan con distintas maneras de entender el mundo, la vida de otros y las relaciones entre las personas.

La educación intercultural pretende ser diferente a la llamada educación multicultural y superador de esta, pues busca la solución a la gestión de la diversidad cultural haciendo énfasis en la educación como transformación y la educación antirracista (Soto-Molina, 2008). Entendido así, proponemos que la comunicación intercultural también es un elemento importante en el ámbito escolar, sobre todo en tiempos de profundos cambios demográficos debido a los procesos migratorios que por distintos motivos se evidencian en todo el mundo. Esta se vincula a temas esencialmente que promueven la inclusión en la escuela con base en la diferencia cultural, por lo que se operativiza a partir de elementos relacionados con el paradigma de la educación intercultural. Además, la comunicación intercultural implica todo un conjunto de variables básicas necesarias para facilitar la mejora de la convivencia escolar. Por ejemplo, se trata de fomentar en el contexto escolar una serie de habilidades para la escucha, la comprensión y la potenciación de la comunicación interpersonal (Leiva-Olivencia \& Márquez-Pérez, 2012).

En el marco de nuestra investigación cobra importancia la perspectiva que nos ofrece la comunicación intercultural en tanto se generan conexiones significativas con respecto al contexto donde se desarrolla el estudio, a saber; escuelas rurales del sur de Chile que poseen un importante número de estudiantes que declaran ser parte de la cultura mapuche huilliche. Justamente en los procesos formativos, la doble configuración de la comunicación intercultural permite establecer diálogos representativos entre dos perspectivas del mundo, las cuales podrían definirse como la indígena y la no 
indígena, lo que además representan un espacio de negociación, que debe tender a ser un espacio de cooperación, para acabar siendo simplemente un espacio de humanización.

Una comprensión más amplia de la conjunción de estos dos campos supondría entrar en el estudio de los procesos hegemónicos en los cuales se debaten nuestras sociedades contemporáneas, en donde las diferencias permean el discurso de la multiculturalidad (Dietz, 2012). Un territorio donde apenas hoy la educomunicación y la interculturalidad comienzan a proponerse como alternativa para ir cerrando las brechas que hoy, más que nunca, evidencian las relaciones humanas. La gran tarea está apenas comenzando.

\section{Bibliografía}

Andrade-Martínez, C. (2020). La educomunicación de Don Bosco y la formación de universitarios como buenos ciudadanos. Estudios Pedagógicos, 46(3), 7-19. https://doi.org/10.4067/S0718-07052020000300007

Aparici, R. (2010). Introducción: la educomunicación más allá del 2.0. En Aparici, R. (Coord.), Educomunicación: más allá del 2.0. (pp. 9-23). Gedisa.

Ari, A., \& Stöckli, A. (2021). Switzerland Case as an Example of Success in Multicultural Education. International Journal of Instruction, 14(2), 1-3. https://bit.ly/3gv72LY

Arispe, V. (2020). Educación intercultural: La perspectiva de los pueblos indígenas de Bolivia. Caracol, (20). https://doi.org/10.11606/issn.2317-9651. i20p166-187

Barbas-Coslado, Á., \& Ortiz de Zárate, A.M. (2020). Vidas de activismo. La participación en los medios de comunicación comunitarios como fuente de aprendizajes. Diálogo Andino, (62), 65-75. https://bit.ly/2S6aDWT

Buckingham, D. (2003). Educación en medios. Alfabetización, aprendizaje y cultura contemporánea. Paidós.

Cebrián de la Serna, M. (2009). Las Tecnologías de la Información y la Comunicación para el desarrollo educativo de los pueblos indígenas. Publicaciones GTEA.

Collado-Ruano, J., Ojeda, M.N., Malo, M.O., \& Amino, D.S. (2020). Educación, artes e interculturalidad: El cine documental como lenguaje comunicativo y tecnología innovadora para el aprendizaje de la metodología I+D+I. Texto Livre, 13(3), 376-393. https://doi.org/10.35699/19833652.2020.25639 
de la Noval-Bautista, L.A. (2018). La radio comunitaria en función del desarrollo. Revista Estudios del Desarrollo Social: Cuba y América Latina, 6(2), 52-64. https://bit.ly/3vb5z1x

de Oliveira Soares, I. (2009). The edu-communication roads: utopias, confrontations, recognitions. Nómadas, (30), 194-207. https://bit.ly/2QBQ9Vd

de Oliveira Soares, I. (2011). Educomunicação. O conceito, o Professional, a aplicação. Paulinas.

Delbury, P. (2020). ¿Racismo en la educación inclusiva? Una mirada desde la interculturalidad crítica. Revista Electrónica Educare, 24(1). https://doi. org/10.15359/ree.24-1.22

Di Caudo, M.V., Llanos-Erazo, D., \& Ospina-Alvarado, M.C. (2016). Interculturalidad y educación desde el Sur. Contextos, experiencias y voces. Universidad Politécnica Salesiana. https://bit.ly/3dBesvi

Dietz, G. (2012). Multiculturalismo, interculturalidad y diversidad en educación: una aproximación antropológica. FCE.

Escarbajal de Haro, A., Escarbajal-Frutos, A., \& García-Martínez, A.J. (2007). La interculturalidad. Desafio para la educación. Dykinsonn.

Esquirol, J. M. (2005). Uno mismo y los otros. De las experiencias existenciales a la interculturalidad. Hender.

Ferraretto, L.A., \& Morgado, F. (2020). Diez pasos para la educación de emergencia por la radio en tiempos de Covid-19. Válega.

Franky, A.P., \& Chiappe, A. (2018). Famílias que educam em casa com TIC: Um estudo qualitativo de múltiplos casos. Ensaio, 26(101), 1324-1346. https:// doi.org/10.1590/S0104-40362018002601507

Gajardo-Carvajal, Y., \& Mondaca-Rojas, C. (2020). Oralidad andina y educación intercultural en zona de frontera, Norte de Chile. Interciencia, 45(10), 488-492. https://bit.ly/3ryxXZ8

García-Canclini, N. (2004). Diferentes, desiguales y desconectados. Mapas de la interculturalidad. Gedisa.

Gibbs, G. (2012). El análisis de datos cualitativos en investigación cualitativa. Morata.

González, F.D.F., \& Rodríguez, N. M. (2019). Teoría decolonial y estudios sobre hábitat: construcción de un soporte epistemológico desde el enfoque de comunicación e interculturalidad. Questión (63). https://bit.ly/31QjZkq

Granda-Merchán, S. (2016). Estado, educación y pueblos indígenas en los Andes ecuatorianos. Alteridad, 11(1), 221-230. https://bit.ly/3dBf7gg

Hernández Díaz, G. (2007). Educomunicación. Desarrollo del pensamiento desde una interdisciplina emergente. Comunicación: Estudios venezolanos de comunicación, (138), 68-78. https://bit.ly/39r1b62 
Hernández-Reyna, M., \& Castillo-Cocom, J.A. (2021). "Ser o no ser indígena": Oscilaciones identitarias dentro de la interculturalidad de Estado en México. Journal of Latin American and Caribbean Anthropology. https:// doi.org/10.1111/jlca.12532

Hidalgo-Santomaro, M.J., \& Gamboa-Chiriboga, C.D. (2012). Campaña comunicativa radiofónica" Regístrate” para la difusión y concienciación de los derechos humanos que poseen refugiados en el Ecuador. Universidad Politécnica Salesiana. https://bit.ly/31ZZO3r

Jackiw, E., \& Haracemiv, S.M.C. (2021). Educomunicação e alfabetização midiática: diálogos freireanos na América Latina. Praxis Educativa, 16, e2116614. https://doi.org/10.5212/PraxEduc.v.16.16614.031

Kaplún, G. (2006). La calle ancha de la comunicación latinoamericana. Educomídia, alavanca da cidadania. UNESCO UMESP. https://bit.ly/3h5fhyP

Laor, T. (2020). Milestones in the development of educational radio in Israel. Israel Affairs, 26(5), 716-738. https://doi.org/10.1080/13537121.2020.1806692

Leiva-Olivencia, J.J., \& Márquez-Pérez, M. (2012). La comunicación intercultural: una herramienta de inclusión en los contextos educativos de diversidad cultural. Revista de Pedagogía, 33(93), 71-93. https://bit.ly/3vJuB8u

Lotero-Echeverri, G., Romero-Rodríguez, L.M., \& Pérez-Rodríguez, A. (2019). Research trends in specialist publications from the field of educommunication and media literacy in latin america. Interface: Communication, Health, Education. 23, e180193 https://doi.org/10.1590/Interface.180193

Marín-Gutiérrez, I., Rivera-Rogel, D., Mendoza-Zambrano, D., \& Zuluaga-Arias, L.I. (2020). Competencia mediática de jóvenes universitarios de Ecuador y Colombia. Trípodos (46), 97-118. https://bit.ly/3xnzkyc

Mata, O. (2010). Didáctica de la Educomunicación. https://bit.ly/3sGelDK

McMillan, J., \& Schumacher, S. (2005). Investigación educativa. Pearson.

Moreira, G. (2020). A educomunicação e os sertões do século XXI. Educacao e Sociedade. 41, e221403, 1-14. http://orcid.org/0000-0002-7752-7607

Muñoz-Borja, P., Sarria, J.M.E., García-Ruiz, R., \& Aguaded, I. (2021). Educomunicación inclusiva y discapacidad en la región andina: revisión cualitativa de avances y logros. Revista Complutense de Educación, 32(1), 67-78. https://doi.org/10.5209/RCED.68017

Núñez-Ruiz, G., Martos-García, A., \& Núñez-Molina, G. (2020). Interculturalidad y educación lingüística del alumnado inmigrante de la provincia de Almería: Un estudio de caso. Porta Linguarum, (34), 209-224. https://doi. org/10.30827/portalin.v0i34.16741 
Parra-Alvarracín, G. (2000). Bases epistemológicas de la educomunicación (Definiciones y perspectivas de su desarrollo). Abya-Yala.

Pérez-Serrano, G. (2016). Investigación cualitativa. Retos e interrogantes. Muralla. Rodríguez-Pastene, F., Niklander Ribera, S., Ojeda, G., \& Vera, E. (2020). Interculturalidad y representación social: el conflicto de la Araucanía en la prensa chilena. Casos Melinao y Luchsinger-Mackay. Estudios sobre el Mensaje Periodístico, 26(4), 1583-1598. https://doi.org/10.5209/ESMP.67388

Sabariego, M., Dorio, I., \& Massot, I. (2019). Metodología de la investigación educativa. En R. Bisquera (Coord.), Metodología cualitativa (pp. 276-366). Arco.

Simons, H. (2011). El estudio de caso: teoría y práctica. Ediciones Morata.

Soto-Molina, J. (2008). El currículo Intercultural bilingüe. La naturaleza humana integrada a su mundo cultural. Magisterio.

Tubino, F. (2005). Las prácticas discursivas sobre la interculturalidad en el Perú de hoy. Propuesta delineamientos para su tratamiento en el sistema educativo peruano. Ministerio de Educación. 